\title{
ON THE PROPERTIES OF A SPECIAL FUNCTION DEFINED BY AN INTEGRAL
}

\author{
M. Emin Erdoğan and C. Erdem İmrak \\ Faculty of Mechanical Engineering, Istanbul Technical University, 34439 \\ Gumussuyu, Istanbul, Turkey. imrak@itu.edu.tr
}

\begin{abstract}
The properties of a special function which is defined by an integral is presented. The numerical values of this function are tabulated correct to twenty decimal places. The curves of this function and its complementary are plotted. Some properties of this function are investigated.
\end{abstract}

Keywords- A function defined by integral, linear differential equation, power series solution, a function defined by a differential equation, tanh function.

\section{INTRODUCTION}

Two classes of nonelementary function are very important in applied mathematics. The first class consists of function arising in connection with linear differential equations. These functions are obtained by power series solutions of linear differential equations. Examples of these functions are Bessel, modified Bessel, Legendre, associated Legendre, Laguerre functions. The second class consists of function defined by integrals which cannot be calculated in terms of finitely many elementary functions. Examples are the Gamma function, the Beta function, the error function, the Exponential integral, the sine and cosine integrals, the Fresnel integral, the Dawson integral. These two class functions are tabulated [1]. Some suggestions have been proposed on the symbols of these functions [2]. The discussions on the properties of these functions may be found in [3].

In this paper, a new function defined by an integral is presented. This function arises due to the calculation of the volume flux for a flow over a plane wall bounded by two side walls perpendicular to the plane. This flow may be a simple but not being realistic model for a paint brush and has been discussed in [4]. The shear stress at the bottom wall cannot be calculated by the expression of the velocity which is in a series form. The reason is that there is a discontinuity in the expression of the velocity, therefore, term-by-term differentiation does not give a convergent series [4]. In order to overcome this difficulty the solution of the governing equation is obtained by the sine transform method. The expressions of the velocity, the volume flux and the stress at the bottom wall have been given in [5].

The expression of the volume flux for the flow over a plane wall bounded by two side walls perpendicular to the plane suggests a function which is in the following form

$$
\int_{0}^{x t-\tanh t} \frac{t^{-3}}{t} t
$$


The origin of this function is discussed and the numerical values are tabulated. The expression of this function for small and large values of the argument are given in a series form in terms of the Bernoulli numbers. The differential equation satisfied by this function is obtained. The curves of this function and its complementary are plotted.

\section{THE ORIGIN OF $F(x)$ FUNCTION}

Assume that the fluid is over a plane wall and between two side walls perpendicular to the plane and that the side walls to be of infinite extent in the $x$ - and $z$ directions as shown in Figure 1. The fluid is set in motion by a constant speed of the bottom wall in the absence of an imposed pressure gradient. The governing equation is

$$
\frac{\partial^{2} u}{\partial y^{2}}+\frac{\partial^{2} u}{\partial z^{2}}=0
$$

where $u$ is the velocity in the $x$ - direction, and $y$ and $z$ are coordinates. It is convenient to take axes fixed in the plates. The boundary conditions become

$$
\begin{array}{lll}
u(y, 0)=U & \text { for } & -b<y<b, \\
u(y, \infty)=0 & \text { for } & -b \leq y \leq b, \\
u( \pm b, z)=0 & \text { for } & 0<z \leq \infty
\end{array}
$$

where $2 b$ is the distance between two side walls.
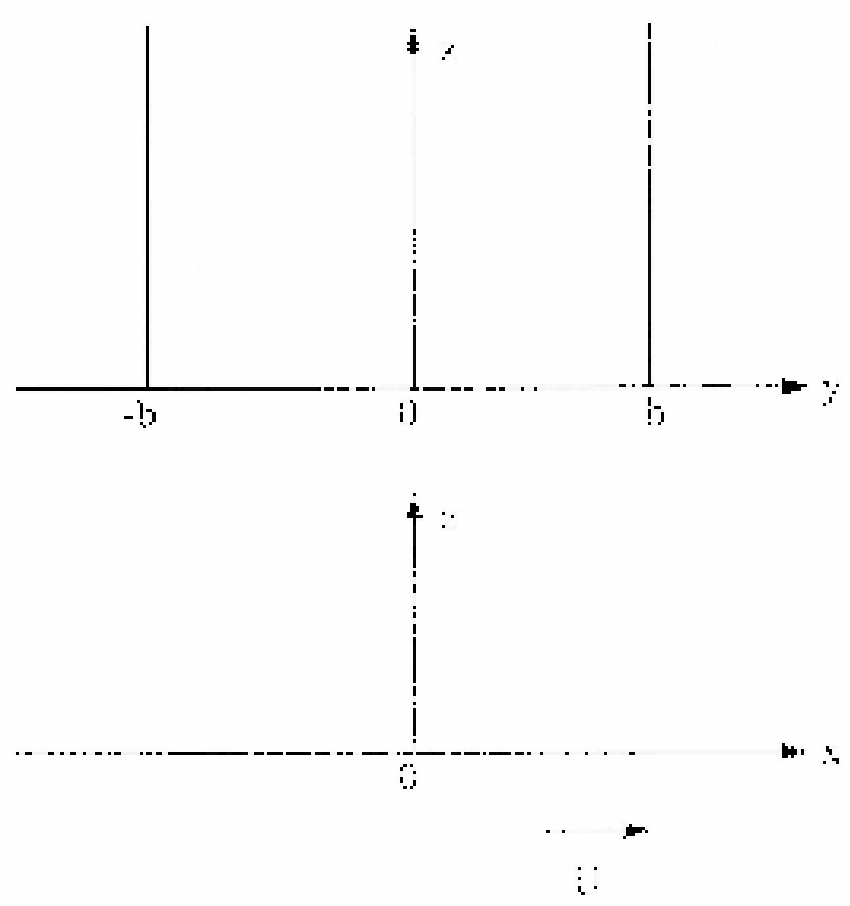

Figure 1. Flow geometry and coordinate system 
The solution subject to the boundary conditions given by (3) is

$$
\frac{u}{U}=\frac{4}{\pi} \sum_{n=0} \frac{(-1)^{n}}{2 n+1} e^{-\lambda_{n} z} \cos \lambda_{n} y
$$

The volume flux is

$$
Q=\int_{-b 0}^{b} \int_{0}^{\infty} u d y d z
$$

Inserting (4) into the expression for $Q$, the volume flux becomes

$$
\frac{Q}{U b^{2}}=\frac{32}{\pi^{3}} \sum_{n=0} \frac{1}{(2 n+1)^{3}} .
$$

The summation in the right-hand side of (5) is tabulated correct to twenty decimal places [1].

The shear stress at the bottom wall cannot be calculated by (4) [5]. The reason is that there is a discontinuity in $u(y, z)$ at $y= \pm b$ and therefore term-by-term differentiation does not give a convergent series. In order to overcome this difficulty, a convenient expression for velocity must be found. This can be realized by applying the sine transform to (2). The sine transform of $u(y, z)$ is [6]

$$
\bar{u}=\int_{0}^{\infty} u \sin \lambda z d z
$$

and the boundary condition becomes $\bar{u}( \pm b)=0$. The application of the sine transform to (2) gives $\bar{u}^{\prime \prime}-\lambda^{2} \bar{u}=-\lambda U$, where a prime denotes differentiation with respect to $y$. The solution subject to the boundary condition is

$$
\bar{u}=\frac{U}{\lambda}\left(1-\frac{\cosh \lambda y}{\cosh \lambda b}\right)
$$

The inverse of $\bar{u}(y, z)$ is [6]

$$
u(y, z)=\frac{2}{\pi} \int_{0}^{\infty} \bar{u} \sin \lambda z d \lambda
$$

Inserting of $\bar{u}$ into this integral one obtains

$$
\frac{u}{U}=1-\frac{2}{\pi} \int_{0}^{\infty} \frac{\cosh \lambda y}{\cosh \lambda b} \frac{\sin \lambda z}{\lambda} d \lambda
$$

This expression of the velocity gives a correct result for the stress at $z=0$. The shear 
stress can be calculated by (6). The shear stress at $z=0$ is in the following form

$$
\frac{\left(\sigma_{x y}\right)_{z=0}}{\rho U^{2}}=\frac{1}{R \cos \frac{\pi y}{2 b}},
$$

where $R=U b / v$. The effect of the side walls on the flow is minimum at $y=0$ and this effect increases near $y= \pm b$.

The volume flux can also be calculated by inserting (6) into the expression of the volume flux. This gives

$$
Q=\frac{4 U b}{\pi} \int_{0}^{\infty}\left(1-\frac{\tanh \lambda b}{\lambda b}\right) \frac{1}{\lambda^{2}} d \lambda \text { or } \frac{Q}{U b^{2}}=\frac{4}{\pi} F(\infty),
$$

where

$$
F(\infty)=\int_{0}^{\infty} \frac{t-\tanh t}{t^{3}} d t
$$

By using the expression given by (5) one obtains

$$
F(\infty)=\frac{8}{\pi} \sum_{0} \frac{1}{(2 n+1)^{3}}
$$

Since the summation on the right-hand side and $1 / \pi^{2}$ are known [1], $F(\infty)$ becomes 0.85255679763493485979 correct to twenty decimal places. The integration in (7) suggests a function that can be defined as

$$
F(x)=\int_{0}^{x} \frac{t-\tanh t}{t^{3}} d t
$$

This integral can be tabulated by using a numerical integration method. The values of $F(x)$ correct to twenty decimal places are given in Table 1 in the Appendix at the end of this paper.

\section{SOME PROPERTIES OF $F(x)$ FUNCTION}

The function $F(x)$ is defined by an integral which is given in (9). The complementary function is defined as

$$
f(x)=\int_{x}^{\infty} \frac{t-\tanh t}{t^{3}} d t .
$$


The integral in (9) can be used for small values of $x$. The integration in (10) can be used for large values of $x$. It is clearly seen that $F(0)=0$ and if one puts $-x$ for the upper limit of the integral in (9), then, one finds $F(-x)=-F(x)$. This shows that $F(x)$ is an odd function of $x$. However, there is no a physical meaning for negative values of the argument. The variation of $F(x)$ and its complementary function $f(x)$ with $x$ are illustrated in Figure 2.

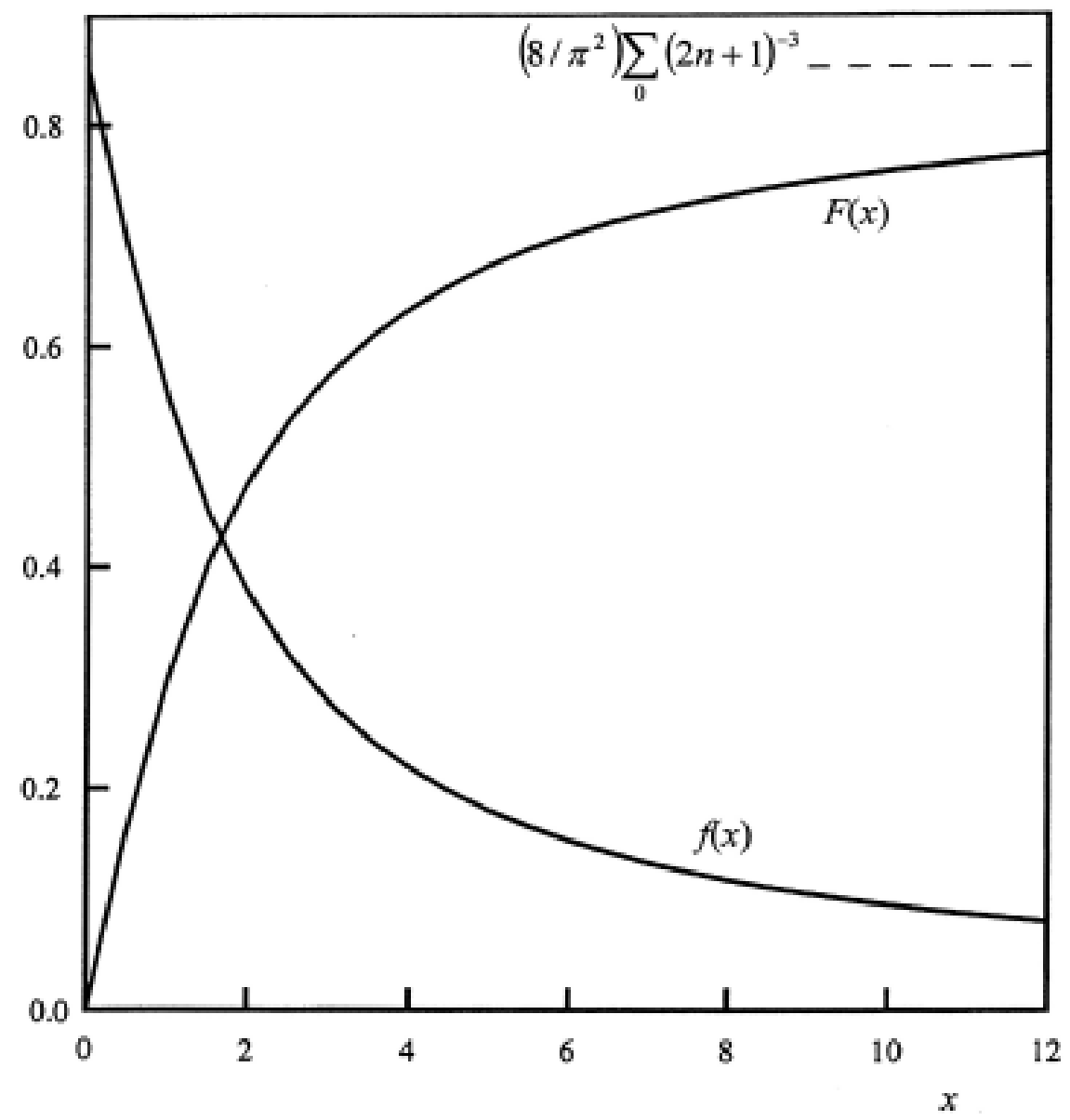

Figure 2. The variations of $F(x)$ and its complementary function $f(x)$ with $x$

For small values of $x, \tanh x$ is in the following form

$$
\begin{aligned}
\tanh x & =\sum_{n=1} \frac{2^{2 n}\left(2^{2 n}-1\right)}{(2 n) !} B_{2 n} x^{2 n-1}, \\
& =x-\frac{x^{3}}{3}+\frac{2 x^{5}}{15}-\frac{17 x^{7}}{315}+\cdots,
\end{aligned}
$$

where $B_{n}$ are the Bernoulli numbers and the generation function is [1] 


$$
\frac{x}{e^{x}-1}=\sum_{n=0} \frac{B_{n} x^{n}}{n !}
$$

and some of them are given in Table 2 in the Appendix at the end of this paper. Inserting the expression of $\tanh x$ into the integral for $F(x)$, one finds

$$
\begin{gathered}
F(x)=\sum_{n=2} \frac{2^{2 n}\left(2^{2 n}-1\right)}{(2 n) !} \frac{B_{2 n} x^{2 n-3}}{(2 n-3)}, \\
=\frac{x}{3}-\frac{2}{45} x^{3}+\frac{17}{1575} x^{5}-\frac{62}{19845} x^{7}+\frac{2764}{1403325} x^{9}-\cdots .
\end{gathered}
$$

This series can be used for $x<1$.

For large values of $x,(9)$ is written in the following form

$$
F(x)=F(\infty)-\frac{1}{x}+\int_{x}^{\infty} \frac{\tanh t}{t} d t
$$

For large values of $x, \tanh x$ is written in the following form

$$
\tanh x=1+2 \sum_{n=1}(-1)^{n} e^{-2 n x}
$$

Inserting this expression into the integral in (12) one obtains

$$
\begin{gathered}
F(x)=F(\infty)-\left(\frac{1}{x}-\frac{1}{2 x^{2}}\right) \\
+2 \sum_{n=1}(-1)^{n} e^{-2 n x}\left(\frac{1}{2 n x^{3}}-\frac{3}{4 n^{2} x^{4}}+\frac{3}{2 n^{3} x^{5}}-\frac{15}{4 n^{4} x^{6}}+\cdots\right) .
\end{gathered}
$$

For large values of $x, F(x)$ can also be written in the following form

$$
F(x)=F(\infty)-\left(\frac{1}{x}-\frac{1}{2 x^{2}}\right)+2 \sum_{n=1}(-1)^{n}\left[e^{-2 n x}\left(\frac{1}{2 x^{2}}-\frac{1}{n x}\right)+2 n^{2} \operatorname{Ei}(2 n x)\right]
$$

where

$$
\operatorname{Ei}(x)=\int_{x}^{\infty} \frac{e^{-t}}{t} d t
$$

is a tabulated function [1]. The series given by (14) can be used for $x>2$. The expression of $F(x)$ given by (14) seems to be more convenient than that of given by (13). 
The function $F(x)$ satisfies the differential equation

$$
x F^{\prime \prime}+3 F^{\prime}=\frac{\tanh ^{2} x}{x^{2}},
$$

with the conditions

$$
F(0)=0 \quad \text { and } \quad F^{\prime}(0)=\frac{1}{3}
$$

where a prime denotes differentiation with respect to $x$. The solution of (15) subject to the condition given by (16) can be found by integrating (15). One of the solution subject to conditions (16) is $F^{\prime}=(x-\tanh x+B) / x^{3}$ with $B=0$, and finally one finds

$$
F(x)=\int_{0}^{x} \frac{t-\tanh t}{t^{3}} d t
$$

\section{CONCLUSIONS}

A new special function which is defined by an integral is presented. This function arises due to the calculation of the volume flux for a flow over a plane wall between two adjoining plates perpendicular to the plane. Some properties of this function are given. The numerical values of this function are tabulated correct to twenty decimal places in the Appendix at the end of this paper.

\section{REFERENCES}

1. M. Abromowitz and I. Stegun, Handbook of Mathematical Functions, New York, Dover, 1965.

2. S.A. Schelkunoff, Proposed symbols for the modified cosine and exponential integrals, Quart. Appl. Math. 1, 90, 1944.

3. R.W. Oliver, Asymptotics and Special Functions, Academic Press, New York, 1974.

4. G. K. Batchelor, An Introduction to Fluid Dynamics, Cambridge University Press, Cambridge, 1967.

5. M.E. Erdoğan, Effects of the side walls on the flow over a moving plane wall, $J$. Appl Mech. and Engng. 2, 415-432, 1997.

6. C.J. Tranter, Integral Transforms in Mathematical Physics, Methuen, London, 1951. 


\section{APPENDIX}

Table 1. The values of $F(x)$

\begin{tabular}{cc|cc}
\hline$x$ & $F(x)$ & $x$ & $F(x)$ \\
\hline 0.0 & 0.00000000000000000000 & 3.6 & 0.61334767992958860727 \\
0.1 & 0.03328899651407222354 & 3.7 & 0.61880073838432503308 \\
0.2 & 0.06631452558705507039 & 3.8 & 0.62401824670277117324 \\
0.3 & 0.09882556412891164988 & 3.9 & 0.62901454757802999218 \\
0.4 & 0.13059454233823164504 & 4.0 & 0.63380290078925494157 \\
0.5 & 0.16142578134896978792 & 4.1 & 0.63839557815887748582 \\
0.6 & 0.19116072601650682672 & 4.2 & 0.64280394784639849348 \\
0.7 & 0.21967989480314026318 & 4.3 & 0.64703855098646190225 \\
0.8 & 0.24690193660146536603 & 4.4 & 0.65110917134018730934 \\
0.9 & 0.27278047904606039786 & 4.5 & 0.65502489858336243600 \\
1.0 & 0.29729955759430265472 & 4.6 & 0.65879418581455240211 \\
1.1 & 0.32046836550855728465 & 4.7 & 0.66242490181912949687 \\
1.2 & 0.34231592206499222852 & 4.8 & 0.66592437858361686054 \\
1.3 & 0.36288607863626382022 & 4.9 & 0.66929945451008410195 \\
1.4 & 0.38223311265042231311 & 5.0 & 0.67255651374120506425 \\
1.5 & 0.40041802136287063529 & 6.0 & 0.69977899682277855398 \\
1.6 & 0.41750552848953442764 & 7.0 & 0.71990373439507604481 \\
1.7 & 0.43356175448656536497 & 8.0 & 0.73536929744851120549 \\
1.8 & 0.44865246826435956073 & 9.0 & 0.74761852601206624236 \\
1.9 & 0.46284182592286171598 & 10.0 & 0.75755679763319982989 \\
2.0 & 0.47619150315818414843 & 11.0 & 0.76577993813068745421 \\
2.1 & 0.48876013646196958540 & 12.0 & 0.77269568652385833829 \\
2.2 & 0.50060300009319802506 & 13.0 & 0.77859230059360817755 \\
2.3 & 0.51177185841530081767 & 14.0 & 0.78367924661474086534 \\
2.4 & 0.52231494513495169141 & 15.0 & 0.78811235319108940978 \\
2.5 & 0.53227703149715988040 & 16.0 & 0.79200992263502900670 \\
2.6 & 0.54169955435349870232 & 17.0 & 0.79546337202944839007 \\
2.7 & 0.55062078223489563378 & 18.0 & 0.79854445195601320151 \\
2.8 & 0.55907600329712348053 & 19.0 & 0.80131026023886464671 \\
2.9 & 0.56709772347057152420 & 20.0 & 0.80380679763500229029 \\
3.0 & 0.57471586656732176479 & 30.0 & 0.81977901985775680771 \\
3.1 & 0.58195797067891152921 & 40.0 & 0.82786929328061920064 \\
3.2 & 0.58884937711841756069 & 50.0 & 0.83275679218882536770 \\
3.3 & 0.59541340957056876348 & 100.0 & 0.84260678676000677623 \\
3.4 & 0.60167154213177220790 & $\infty$ & 0.85255679763493485979 \\
3.5 & 0.60764355564695327061 & & \\
\hline & & & \\
\hline
\end{tabular}

Table 2. Some of the Bernoulli numbers [1].

\begin{tabular}{cc|cc|cc|cc}
\hline$n$ & $B_{n}$ & $n$ & $B_{n}$ & $n$ & $B_{n}$ & $n$ & $B_{n}$ \\
\hline 0 & 1 & 4 & $-1 / 30$ & 8 & $-1 / 30$ & 12 & $-691 / 2730$ \\
1 & $-1 / 2$ & 5 & 0 & 9 & 0 & 13 & 0 \\
2 & $1 / 6$ & 6 & $1 / 42$ & 10 & $-5 / 56$ & 14 & $7 / 6$ \\
3 & 0 & 7 & 0 & 11 & 0 & 15 & 0 \\
\hline
\end{tabular}

